\title{
Evidence for oxidative stress in unstable angina
}

\author{
John McMurray, Mridula Chopra, Ibrahim Abdullah, W Ewen Smith, Henry J Dargie
}

Department of Cardiology, Western Infirmary, Glasgow

J McMurray

I Abdullah

H J Dargie

Department of Chemistry, University of Strathclyde, Glasgow

M Chopra

W E Smith

Correspondence to: Dr John McMurray Department of Cardiology, Western Infirmary, Glasgow G11 6NT.

Accepted for publication 11 May 1992.

\begin{abstract}
Objective-To determine whether unstable angina, which is characterised by recurring episodes of myocardial ischaemia and reperfusion, is associated with oxidative stress (that is, where there is an imbalance between oxidants, such as free radicals, which are in excess and antioxidants).
\end{abstract}

Design-Between group comparison of patients with unstable angina, stable angina, and healthy controls.

Setting-The coronary care unit and cardiac investigation ward of a regional cardiology centre.

Patients-Twenty five consecutive patients admitted to the coronary care unit with unstable angina. Twenty five consecutive patients admitted to the cardiac investigation ward (patients with stable angina undergoing coronary angiography) were used as controls for the presence of atherosclerosis, drug treatment, and smoking habit. Thirty eight healthy controls (hospital staff and patients admitted for minor surgical procedures who were otherwise healthy) were also studied.

Main outcome

measures-Thiobarbituric acid related substances (TBARS) in plasma and plasma reduced thiol (PSH) as indicators of oxidative damage to lipids and proteins respectively were measured. Coronary angiography was performed in all patients with stable angina and roughly half of those with unstable angina.

Results-Mean (SEM) plasma TBARS in unstable angina and stable angina were $9.95(0.36) \mathrm{nmol} / \mathrm{ml}$ and $9.14(0.28) \mathrm{nmol} /$ ml respectively $(p=0 \cdot 08)$. Mean plasma TBARS in healthy controls were 8.09 $(0.21) \mathrm{nmol} / \mathrm{ml}$ ( $\mathrm{p}<0.05$ compared with both angina groups). Mean (SEM) PSH concentration in unstable angina was $4 \cdot 21$ (9) $\mathrm{nmol} / \mathrm{ml}$ and in stable angina was 4.85 (9) $\mathrm{nmol} / \mathrm{ml}$ (p < 0.05). Mean PSH in healthy controls was 5.64 (8) $\mathrm{nmol} / \mathrm{ml}$ ( $p<0.001$ compared with both angina groups). The extent of coronary artery disease, use of medication, and smoking habit were not significantly different between the angina groups.

Conclusions-Biochemical indicators of oxidative stress are more abnormal in unstable than stable angina. This is in keeping with experimental evidence that episodes of ischaemia and reperfusion lead to generation of free radicals and toxic oxygen species and depression of endogenous antioxidant activity. The clinical significance of this finding remains to be determined, although, experimentally, free radicals and toxic oxygen species have adverse effects on myocardial contractile function, myocardial electrical stability, endothelial mediated vasodilatation, and coagulation.

\section{(Br Heart J 1992;68:454-7)}

Free radicals and related toxic oxygen species have been implicated in a growing number of disease processes. Two mechanisms common to these processes are thought to lead to generation of free radicals and toxic oxygen species: these are the sequence of tissue ischaemia followed by reperfusion, and the activation of neutrophil nicotinamide adenine dinucleotide phosphate (NADPH) oxidase and myeloperoxidase in the respiratory burst. ${ }^{1-3}$ Endogenous antioxidant defences may also be depressed in these circumstances. ${ }^{1-3}$ Where there is an imbalance between oxidant (eg, free radical) and antioxidant activity in favour of the oxidant oxidative stress is said to exist. Free radicals and associated toxic oxygen species are highly unstable chemically and immediately react with adjacent biomolecules to cause cellular and tissue damage. ${ }^{1-3}$ In experimental models contractile and vascular endothelial injury can be caused in the heart by free radicals and toxic oxygen species. ${ }^{3-5}$ Clinically, one common condition characterised by recurring episodes of myocardial ischaemia and reperfusion and neutrophil activation is unstable angina. ${ }^{\circ} \mathrm{We}$ have sought biochemical evidence of oxidative stress in patients with unstable angina. Concentrations of indicators of free radical damage to lipids (lipid peroxidation-that is, plasma thiobarbituric acid reactive substances (TBARS)) and proteins (thiol or sulphydryl group oxidation-that is, decreased plasma reduced thiol (PSH)) were measured. ${ }^{17}$ Where there is an imbalance between oxidants (for example, free radicals) and antioxidants in favour of oxidants (where there is oxidative stress), lipid peroxidation is increased and protein thiols are decreased. ${ }^{17}$

\section{Patients and methods \\ PATIENTS}

Unstable angina

Twenty five consecutive patients admitted to the coronary care unit with a confirmed diagnosis of unstable angina were studied. Unstable angina was defined as an increase in frequency or severity or both of existing angina or recent 
onset angina requiring a stay in hospital. ST segment depression or $\mathrm{T}$ wave inversion had to be present on the electrocardiogram, and patients who had a rise in creatine kinase $>150$ iu/l within 24 hours of admission were excluded from analysis. Patients with postinfarct angina were not recruited.

\section{Stable angina}

Twenty five consecutive patients with chronic stable angina and a positive exercise tolerance test (that is one associated with a $>1 \mathrm{~mm} \mathrm{ST}$ segment depression by the end of stage III of the Bruce protocol) were recruited. All of these patients had been admitted for elective cardiac catheterisation under the care of one consultant cardiologist.

This study was approved by the West Ethical Committee of the Greater Glasgow Health Board and all patients gave informed consent before the study.

\section{MEDICATION AND SMOKING HABIT}

Oral and parenteral cardiac medication prescribed at the time of study were recorded. Smoking habit was also recorded.

\section{CARDIAC CATHETERISATION}

Patients with unstable angina were catheterised before discharge if their symptoms failed to settle on medical treatment, or if after discharge they had disabling angina despite medical treatment, or a positive exercise tolerance test. The coronary angiograms were reported independently of the biochemical analyses. A stenosis of $>50 \%$ was taken as significant.

\section{NORMAL CONTROLS}

Healthy controls were recruited from the hospital staff and from patients admitted for minor surgical procedures.

\section{BLOOD SAMPLES}

All blood samples were taken at roughly 08.30 hours, after an overnight fast. The samples were taken from the patients with unstable angina the first morning after admission, and from the stable angina patients the morning after admission but before catheterisation. Samples were taken from an antecubital vein into chilled lithium heparin tubes and centrifuged immediately at $5 \cdot 0^{\circ} \mathrm{C}$. Plasma was separated and analysed the same day.

Figure 1 Plasma concentrations of thiobarbituric acid related substances

(malondialdehyde $(M D A)$ ) in healthy controls (normal), stable angina patients, and patients with unstable angina. Individual values are given with mean value indicated by a horizontal bar. ${ }^{\star}, p<0.02$ between angina groups and normal. Bracket denotes statistical comparison of the two angina groups.

\section{BIOCHEMICAL ASSAYS}

Plasma concentrations of TBARS (malondialdehyde, MDA) were measured as already described. ${ }^{8}$ Spectrophotometrical measurements of PSH were made with Ellmans reagent. The within and between assay coefficients of variance for these assays were: malondialdehyde $5 \%$ within and $6 \%$ between assays; PSH $2 \%$ within and $3 \%$ between assays.

\section{STATISTICAL ANALYSIS}

An unpaired $t$ test was used to compare the three groups with Bonferroni's correction for multiple comparisons.

\section{Results \\ CLINICAL CHARACTERISTICS \\ Healthy controls}

Thirty eight controls (16 men) aged 21 to 65 years were recruited. None was on regular medication. Ten were current smokers.

\section{Unstable angina}

Patients aged 32 to 80 (mean 57 (SEM 2)) years, 15 men and 10 women were recruited. Nine were current smokers. In $80 \%$ treatment was with a $\beta$ blocker, $76 \%$ with a nitrate, and $44 \%$ with a calcium channel blocker. Aspirin was taken by $84 \%$ and heparin by $28 \%$. One patient eceived streptokinase and one patient had ventricular fibrillation (neither had had an infarction). One patient had a myocardial infarction more than 24 hours after admission. Twelve patients had a past myocardial infarction. Thirteen patients $(52 \%)$ underwent cardiac catheterisation. Single vessel disease was present in $23 \%$ double vessel disease in $15 \%$, and triple vessel disease in $46 \%$; four ( $16 \%$ ) patients had had coronary artery bypass grafting.

\section{Stable angina}

Results were analysed for 20 men and four women (one women patient had normal coronary arteries) aged 38-70 (mean (SEM) 58 (2)) years. Twelve patients were current smokers. The treatment for $71 \%$ was with a $\beta$ blocker, $67 \%$ with a nitrate, $71 \%$ with a calcium channel blocker, and $42 \%$ were taking aspirin. Five patients had a past myocardial infarction. Twenty nine per cent of patients had single vessel disease, $13 \%$ double vessel disease, and $42 \%$ triple vessel disease; four $(17 \%)$ had had coronary artery bypass grafting.

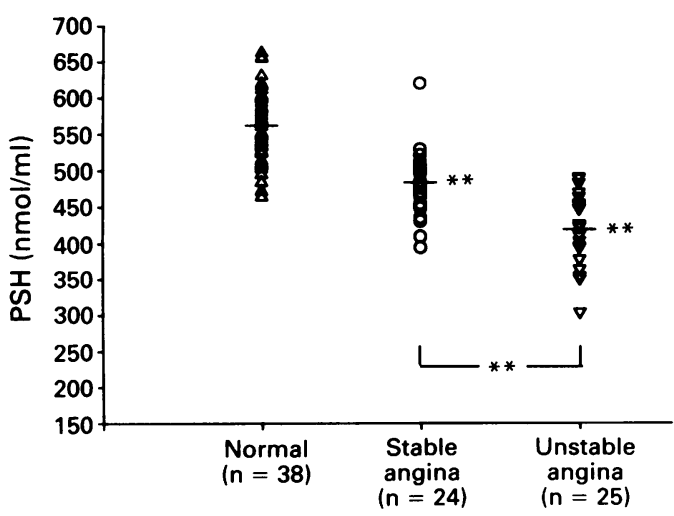

Figure 2 Plasma thiol concentration (PSH) in each of the three groups as shown in fig $1 .{ }^{\star \star}, p<0.01$ for comparisons as in fig 1. 
BIOCHEMICAL MEASURES OF OXIDATIVE STRESS TBARS (malondialdehyde)

Both groups of angina patients had significantly higher plasma malondialdehyde concentrations than the normal controls (fig 1). Mean plasma malondialdehyde seemed to be higher in patients with unstable angina compared with those with stable angina although this difference did not reach significance $(p=0.08)$.

\section{PSH}

In both groups of angina patients compared with controls PSH was significantly lower (fig 2 ), and PSH was also significantly lower in patients with unstable angina compared with those with stable angina (fig 2).

\section{Discussion}

Both groups of patients with coronary artery disease had abnormal indicators of oxidative stress in keeping with previous findings. ${ }^{910}$ Interestingly, however, this abnormality was greatest in those patients with symptomatically overt ischaemia and could not be explained by such differences as extent of coronary disease or medication. This is in keeping with the belief that ischaemia and reperfusion, or neutrophil activation, or both cause generation of free radicals and other oxidants. Similar abnormalities in both markers measured in this study were noted in another study of myocardial ischaemia and reperfusion. In that study, Davies $e t$ al noted a rise in TBARS and a fall in PSH in patients with myocardial infarction who successfully reperfused after thrombolytic treatment. ${ }^{112}$

In our study the concentrations of the biochemical measures showed considerable overlap. This may reflect the fact that even stable angina patients have frequent episodes of recurring ischaemia (symptomatic or silent) and this may reduce the biochemical differences between the two angina groups. Decreased PSH has also been found in patients with stable angina and acute myocardial infarction. ${ }^{913}$ Similarly, TBARS are increased after pacing induced ischaemia and during percutaneous transluminal coronary angioplasty in humans. ${ }^{14} 15$

What might the relevance of these findings be? It is known that abnormalities of wall motion may persist for days to weeks after an episode of unstable angina. ${ }^{16}$ Such dysfunction seems to be the clinical correlate of the reversible experimental mechanical reperfusion injury that is apparently caused by the generation of free radicals and oxidants. ${ }^{16}$ Oxidative stress may also provoke the arrhythmias that occur in a few patients with unstable angina (ventricular fibrillation occurred in one patient in this study). ${ }^{45}$ Lipid peroxides, products of free radical damage to lipids, may cause damage to endothelial cells, decrease generation of vascular prostacyclin, and promote thrombosis. ${ }^{510}$ All of these effects are potentially relevant and disadvantageous in patients with unstable angina.

\section{LIMITATIONS}

There are several limitations to this study. Methods of measuring lipid peroxides using thiobarbituric acid have been criticised on the basis of specificity. Nevertheless, this type of test remains an extremely sensitive one and is the most commonly used biochemical measure of oxidative stress. Abnormalities detected by us with the particular method used in this study have previously correlated well with other measures of redox state such as glutathione and superoxide dismutase; ${ }^{17}$ also another marker, $\mathrm{PSH}$, was used in this study to corroborate free radical damage.

A second limitation is that only a proportion of patients with unstable angina underwent cardiac catheterisation. It may therefore be argued that the extent of coronary disease in the whole unstable group could be greater than in the stable group. This would mean, however, that those in the unstable group who were not catheterised would have to have had more extensive coronary disease than those who were catheterised. This is highly unlikely as in our unit catheterisation is performed on the basis of either $(a)$ failure to respond symptomatically to treatment or $(b)$ the finding of a positive exercise tolerance test on medical treatment. It is probable, therefore, that those in the unstable group who were not catheterised had less rather than more extensive coronary disease. Thus it seems unlikely that the addition of noncatheterised unstable patients could have imbalanced the groups in terms of extent of coronary artery disease, in a way that would invalidate the results.

In summary we have shown that unstable angina is associated with abnormal plasma biochemical markers of oxidative stress. These findings support several recent findings in patients with other forms of myocardial ischaemia and reperfusion. The clinical relevance of these findings cannot be determined from this preliminary study. Unstable angina is, however, often associated with mechanical dysfunction and thrombotic coronary occlusion, both potential consequences of the generation of free radicals and toxic oxygen species. An association between abnormally high concentrations of indicators of oxidative stress and ventricular dysfunction or the occurrence of myocardial infarction would strengthen the case that biological oxidants including free radicals are clinically important.

$\mathrm{J} \mathrm{McM}$ is a British Heart Foundation Intermediate Research Fellow. M C was supported by a grant from the Bristol MyersSquibb Company.

1 Lunec J. Free radicals: their involvement in disease processes. Ann Clin Biochem 1990;27:173-82.

2 Dormandy TL. Free radical pathology and medicine. Journal of the Royal College of Physicians of London 1989;23:221-7.

$3 \mathrm{McCord}$ JM. Oxygen derived free radicals in post ischemic tissue injury. N Engl J Med 1985;312:159-63.

4 Kloner RA, Przyklenk K, Whittaker P. Deleterious effects of oxygen free radicals in ischemia/reperfusion: resolved and unresolved issues. Circulation 1989;80:1115-27.

5 Forman MB, Puett DW, Virmani R. Endothelial and myocardial injury during ischemia and reperfusion: pathogenesis and therapeutic implications. $\mathrm{J} \mathrm{Am} \mathrm{Coll}$ Cardiol 1989;13:450-9.

6 Dinerman JL, Mehta JL, Saldeen TGP, Emerson S, Wallia R, Darda R, Davidson A. Increased neutrophil elastase 
release in unstable angina pectoris and acute myocardial infarction. J Am Coll Cardiol 1990;15:1559-63.

7 Kosugi H, Kikugawa K. Potential thiobarbituric acid reactive substances in peroxidised lipids. Free Rad Biol Med 1989;7:205-7.

8 McMurray J, McLay J, Chopra M, Bridges A, Belch JJF Evidence for enhanced free radical activity in chronic congestive heart failure secondary to coronary artery disease. Am J Cardiol 1990;65:1261-2.

9 Belch JJF, Chopra M, Hutchison S, Lorimer R, Sturrock $\mathrm{RD}$, Forbes CD, Smith WE. Free radical pathology in chronic arterial disease. Free Rad Biol Med 1989;6:375-8.

10 Stringer MD, Gorog PG, Freeman A, Kakkar W. Lipid peroxides and atherosclerosis. $B M J$ 1989;298:281-4

11 Davies SN, Ranjadayalan K, Wickens DG, Dormandy TL, Timmis AD. Lipid peroxidation associated with successful thrombolysis. Lancet 1990;i:741-3.

12 Davies SW, Wickens DG, Ranjadayalan K, Dormandy TL Timmis AD. Oxidative stress on reperfusion of myocar- dial infarction in man. Clin Sci 1991;80 (suppl 24):25.

13 Kadota K, Yui Y, Yoshiharu M, Takahashi M, Aoyama T, Shirotani $M$, et al. Oxidation of sulfhydryl groups of serum albumin in coronary artery diseases. $\mathrm{J} \mathrm{Am}$ Coll Cardiol 1989;13(suppl A):44.

14 Roberts MJD, Young IS, Trouton TG, Trimble ER, Khan MM, Webb SW, et al. Transient release of lipid peroxides after coronary artery balloon angioplasty. Lancet 1990;ii:143-5.

15 Oldroyd KG, Chopra M, Rankin AC, Belch JJF, Cobbe SM. Lipid peroxidation during myocardial ischaemia induced by pacing. Br Heart J 1990;63:88-92.

16 Kloner RA, Przyklenk K, Patel B. Altered myocardial states: the stunned and hibernating myocardium. Am J Med 1989;86(suppl 1A):14-22.

17 McMurray J, Chopra M, Smith WE, Dargie HJ. Free radical activity in chronic heart failure: evidence for transmyocardial oxidative stress. Circulation 1990; 82(suppl III):566. 